\title{
AUTHOR INDEX (Volume 4)
}

Adler, J. Chang, I. \& Shapira, S. Visualization of graphical methods of series analysis

Adler, S.L. \& Bhanot, G.V. V cycle dynamical exponent of the Multi-Scale-Update algorithm for the 2-d XY model

Adorni, G. \& Conte, G. The connection machine at the University of Parma

Akemi, K. see Stamatescu, I.O.

Alimi, J.-M. \& Scholl, H. Formation of large scale structures of the universe on the connection machine-2

Andreichenko, V.B. see Talapov, A.L.

Andreoni, A. Ab initio theoretical approaches to the struc tural, electronic and vibrational properties of small clusters and fullerenes: the state of the art

Apostolakis, J. Coddington, P. \& Marinari, E. New SIMD algorithms for cluster labeling on parallel computers

B

Baker, M. Kalkreuter, T., Mack, G. \& Speh, M. Neural multigrid methods for gauge theories and other disordered systems

Bali, G.S. see Schilling, $K$.

Bali, G.S. Schilling, K. Fingberg, J., Heller, U.M. \& Karsch, F. Computation of the spatial string tension in high temperature $S U(2)$ gauge theory

Bartoloni, A. Bastianello, G., Battista, C., Cabasino, S. Marzano, F., Paolucci, P.S., Pech, J., Rapuano, F., Panizzi, E., Sarno, R., Todesco, G.M., Torelli, M., Tross, W., Vicini, P., Cabibbo, N. \& Tripiccione, $\mathrm{R}$. The software of the APE100 processor
4 (1993) 1007

4 (1993) 947

4 (1993) 1

4 (1993) 1233

$4(1993) 197$

4 (1993) 787

4 (1993) 227

4 (1993) 749

4 (1993) 239

4 (1993) 1167

4 (1993) 1179

$4(1993) 955$
Bartoloni, A. Bastianello, G. Battista, C., Cabasino, S., Marzano, F., Paolucci, P.S., Pech, J., Rapuano, F., Panizzi, E., Sarno, R., Todesco, G.M., Torelli, M., Tross, W., Vicini, P., Cabibbo, N., Fucci, A. \& Tripiccione, R. A hardware implementation of the APEIOO architecture

Bartoloni, A. Battista, C., Cabasino, S., Paolucci, P.S., Pech, J., Sarno, R., Todesco, G.M., Torelli, M., Tross, W., Vicini, P., Benzi, R., Cabibbo, N., Massaioli, F. \& Tripiccione, R. LBE simulations of Rayleigh-Benard convection on the APE100 parallel processor

Basermann, A. Conjugate gradients parallelized on the hypercube

Bastianello, G. see Bartoloni, A.

Bastianello, G. see Bartoloni, A.

Battista, C. see Bartoloni, A.

Battista, C. see Bartoloni, A.

Battista, C. see Bartoloni, A.

Beazley, D.M. see Lomdahl, P.S.

Bel'kov, A.A. Ebert, D., Lanyov,

A.V. \& Schaale, A. Heat-kernel calculation of quark determinant and computer algebra

Benzi, R. see Succi, S.

Benzi, R. see Bartoloni, A.

Berg, B.A. Multicanonical Monte Carlo simulations

Bernardes, A.T. \& Herrmann, H.J. A simple model with strong asymmetric couplings

Bhanot, G. 3-d spin glasses and ising models - new methods on new architectures

4 (1993) 993

4 (1993) 1295

4 (1993) 955

4 (1993) 969

4 (1993) 955

4 (1993) 969

4 (1993) 993

4 (1993) 1075

4 (1993) 775

4 (1993) 409

4 (1993) 993

4 (1993) 249

$4(1993) 765$

Bhanot, G.V. see Adler, S.L.

Billoire, A. Monte Carlo simulation of a 3-D frustrated Heisenberg model

Bode, A. Lippert, T., Schilling, K. \& Ueberholz, P. Determination of monopole current clusters in four-dimensional quantum electrodynamics 
Bona, C. \& Massó, J. Numerical relativity: Evolving spacetime Bonomi, E. see Fattebert, J.-L.

Booth, S. Assembly language programming for the 1860

Bottcher, C. see Wells, J.C.

Bottcher, C. see Dean, Dw.

Bottcher, C. see Dean, $D_{N} J$

Broggi, A. d'Andrea, V. \&

Destri, G. Cellular automato as a computational model for low-level vision

Buchholtz, V. \& Pöschel, T. A vectorized algorithm for molecular dynamics of shor range interacting particles

Butterlin, N. see Goedgebuer, J.-P.

C

Cabasino, S. see Bartoloni, A Cabasino, S. see Bartoloni, A. Cabasino, S. see Bartoloni, A.

Cabibbo, N. see Bartoloni, A.

Cabibbo, N. see Bartoloni, A

Cabibbo, N. see Bartoloni, A.

Cabral, F. Lago, A. \& Gallas, J.A.C. A picture book of two families of cubic maps

Caffo, M. Czyz, H. \& Remiddi, E. BHAGEN: An event generator of bhabha scattering at $Z$ energy, for small and large scattering angles

Calvo, M.P. see Sanz-Serna, J.M.

Carl, U. see Wang, D.C.

Chaara, A.M. Kr̈oger, H., Moriarty, K.J.M. \& Potvin, J. Glueball and meson dispersion relations in compact $U(1)_{2+1}$ lattice gauge theory

Chang, I. see Adler, $J$.

Chiccoli, C. Pasini, P., Semeria,

F. \& Zannoni, C. An application of a cluster Monte Carlo method to the Heisenber model

Chopard, B. Parallel computing at the University of Geneva

Chopard, B. \& Droz, M. Study of the $A+B \rightarrow C$ reaction-diffusion process

Choy, T.L. \& Yeung, Y.Y. Decoupling transformation ap proach to lattice dynamics in face-centred cubic metals

Christodoulos, F. see Gillan, MJ.

Č́žek, J. Vinette, F. \& Weniger, E.J. On the use of the symbolic language maple in physics and chemistry: several examples
4 (1993) 883 $4(1993) 539$

4 (1993) 1279

4 (1993) 459

4 (1993) 723

4 (1993) 1023

4 (1993) 5

4 (1993) 1049

4 (1993) 299

4 (1993) 955

4 (1993) 969

4 (1993) 993

4 (1993) 955

4 (1993) 969

4 (1993) 993

4 (1993) 553

4 (1993) 591

4 (1993) 385

4 (1993) 151

4 (1993) 919

4 (1993) 1007

4 (1993) 1041

4 (1993) 207

$4(1993) 209$

4 (1993) 493

4 (1993) 287

4 (1993) 257
Coddington, P. see Apostolakis $J$.

$4(1993) 749$

Conte, G. see Adorni, G.

4 (1993) 1

Cosmo, G. \& De Angelis, A. A hybrid neural network architecture for the classification of the hadronic decays of the $Z^{0}$ Czyz, H. see Caffo, $M$.

$4(1993) 977$

4 (1993) 591

D

d'Andrea, V. see Broggi, A. 4 (1993) 5

De Angelis, A. see Cosmo, G. 4 (1993) 977

Dean, D.J. Bottcher, C. \& Strayer, M.R. Spline techniques for solving relativistic conservation equations

Dean, D.J. Bottcher, C., Strayer, M.R. \& Wells, J.C. Parallel implementation of 3+1-dimensional relativistic hydrodynamics

de Forcrand, Ph. see Stamatescu, lo

Destri, G. see Broggi, A.

Dickman, R. Nonequilibrium phase transitions in catalysis and population models

Dotsenko, VI.S. see Talapov, A.L.

Drake, J. see Wells, J.C.

Drouffe, J.-M. see Maggs, A.C.

Droz, M. see Chopard, $B$

E

Ebert, D. see Bel'kov, A.A.

Edelsohn, D.J. Hierarchical treestructures as adaptive meshes

Essafi, H. see Pic, M.M

Esselink, K. see Smit, $B$.

Evertz, H.G. \& Marcu, M. A nonlocal approach to vertex models and quantum spin systems

4 (1993) 1147

F

Fattebert, J.-L. \& Bonomi, E. Isothermal molecular dynamics: A practical study

Ferng, W. see Petiton, S.

Fingberg, J. see Bali, G.S.

Flanery, R. see Wells, J.C.

Form, W. Ito, N. \& Kohring, G.A. Vectorized and parallelized algorithms for multimillion particle $M D$. simulations

4 (1993) 1233

4 (1993) 5

4 (1993) 271

4 (1993) 787

4 (1993) 459

4 (1993) 339

4 (1993) 209

$4(1993) 775$

4 (1993) 909

$4(1993) 97$

4 (1993) 393

Frasconi, P. Gori, M. \& Soda, G. DAPHNE: Data parallelism neural network simulator

Fucci, A. see Bartoloni, A.

Fujisaki, M. see Stamatescu, I.O.
4 (1993) 17

4 (1993) 539

4 (1993) 65

4 (1993) 1179

4 (1993) 459

4 (1993) 1085

4 (1993) 969

4 (1993) 1233 
G

Gallas, J.A.C. see Cabral, F.

Gerdt, V.P. Computer algebra, symmetry analysis and integrability of nonlinear evolution equations

Gerling, R.W. see Jakobs, A.

Gillan, M.J. Christodoulos, F. The path-integral quantum simulation of hydrogen in metals

Goedgebuer, J.-P. Butterlin, N. \& Porte, H. Digital optical computing: a new approach of systolic arrays based on coherence modulation of light and integrated optics technology

Gori, M. see Frasconi, P.

Grassberger, P. On efficient box counting algorithms

Grønbech-Jensen, N. see Lomdahl, P.S.

$\mathbf{H}$

Hackl, R. Matuttis, H.-G., Singer Th. Husslein, J.M. \& Morgenstern, I. Parallelization of the $2 D$ Swendsen-Wang algorithm

Hammarlund, P. Levin, B. \& Lansner, A. Biologically real istic and artificial neural network simulators on the connection machine

Hashimoto, T. see Stamatescu, IO

Hedman, F. \& Laaksonen, A. An approach to data parallel molecular dynamics for liquids

Hege, C. see Stamatescu, I.O.

Heller, U.M. see Bali, G.S

Hemker, A. A data parallel problem solving architecture for the reconstruction of physical events

Herrmann, H.J. Molecular dynamics simulations of granular materials

Herrmann, H.J. see Bernardes, A.T.

Hilbers, P.A.J. see Smit, B.

Hioki, S. see Stamatescu, I.O

Hsu, Y.P. Development of a gaussian hypergeometric function code in complex domains

I

Iori, G. Marinari, E. \& Parisi, G. Heteropolymer folding on a APE-100 supercomputer
$4(1993) 553$

4 (1993) 279

4 (1993) 983

$4(1993) 287$

4 (1993) 299

$4(1993) 17$

4 (1993) 515

4 (1993) 1075

$4(1993) 1117$

$4(1993) 49$

4 (1993) 1233

4 (1993) 41

4 (1993) 1233

4 (1993) 1179

4 (1993) 143

$4(1993) 309$

4 (1993) 765

4 (1993) 393

4 (1993) 1233

$4(1993) 805$

\section{4 (1993) 1333}

Ito, N. see Kawashima, $N$.

$4(1993) 525$

Ito, N. Kikuchi, M. \& Okabe, Y. Recycle of random sequences $\quad 4$ (1993) 569

Ito, N. see Form, $W$.

Ito, N. Parallelization of the ising simulation

4 (1993) 1085

$4(1993) 1131$

J

Jakobs, A. \& Gerling, R.W. Scaling results for parallelism on the intel $i 860$ hypercube

Jauslin, H.R. Numerical implementation of a K.A.M. algorithm

$\mathbf{K}$

Kalkreuter, T. see Baker, $M$.

Kanada, Y. see Kawashima, $N$.

Kanaya, K. QCD calculations on the QCDPAX

Karsch, F. see Bali, G.S.

Karsch, F. Laursen, M.L., Neuhaus, T. \& Plache, B. Fluctuations of Chern-Simons numbers in the SU(2) higgs model.

Kawashima, N. Ito, N. \& Kanada, Y. Algorithms for Monte Carlo simulations of the ising models on a simple cubic lattice

Kenway, R. UKQCD-recent results and future prospects

Kikuchi, M. see Ito, N.

Kleiss, R. Quasi-Monte Carlo, quasi-random numbers and quasi-error estimates

Kohring, G.A. see Form, W.

Kroger, H. see Chaara, A.M.

L

Laaksonen, A. see Hedman, $F . \quad 4$ (1993) 41

Lago, A. see Cabral, F.

Lakhtakia, A. Strong and weak forms of the method of moments and the coupled dipole method for scattering of timeharmonic electromagnetic fields

Lansner, A. see Hammarlund, P. Lanyov, A.V. see Bel'kov, A.A.

Laursen, M.L. see Karsch, F.

Lauwers, P.G. \& Wittlich, T. Inversion of the fermion matrix in lattice $Q C D$ by means of parallel-transported multigrid (PTMG)

4 (1993) 983

4 (1993) 317

4 (1993) 239

4 (1993) 525

4 (1993) 1221

4 (1993) 1179

$4(1993) 1195$

4 (1993) 525

4 (1993) 1255

4 (1993) 569

4 (1993) 323

$4(1993) 1085$

4 (1993) 919

4 (1993) 553

4 (1993) 721

4 (1993) 49

4 (1993) 775

4 (1993) 1195

Leibler, S. see Maggs, A.C.

4 (1993) 609

4 (1993) 339

Levin, B. see Hammarlund, P. $\quad 4$ (1993) 49 
Lippert, T. Lattice quantum electrodynamics near the phase transition

Lippert, T. see Bode, A

Lomdahl, P.S. Beazley, D.M. Tamayo, P. \& GrønbechJensen, N. Multi-million particle molecular dynamics on the CM-5

\section{M}

Mack, G. see Baker, $M$.

Maggs, A.C. Drouffe, J.-M. \& Leibler, S. Physics of fluctuat ing membranes

Mairesse, R. see Moussiaux, A.

Mantica, G. Plato, Apollonius, and Klein: playing with spheres

Marcu, M. see Evertz, H.-G.

Marinari, E. see Apostolakis, J.

Marinari, E. see Iori, G

Marro, J. Steady states in nonequilibrium lattice systems

Marzano, F. see Bartoloni, A.

Marzano, F. see Bartoloni, A.

Massaioli, F. see Succi, S.

Massaioli, F. see Bartoloni, A

Massó, J. see Bona, $C$

Matuttis, H.-G. see Hackl, $R$.

Melin, S. Simulation of sound propagation in granular media on the connection machine

Miyamura, O. see Stamatescu, I.O

Möller, R. see Wang, D.C.

Mora, P. \& Place, D. A lattice solid model for the nonlinear dynamics of earthquakes

Morgenstern, I. see Hackl, R.

Moriarity, K.J.M. \& Trappen-

berg, T. Programming tools for parallel computers

Moriarty, K.J.M. see Chaara, A.M.

Moussiaux, A. Mairesse, $\mathrm{R}$ CONVODE: A REDUCE package for differential equations

Munkel, C. Large scale simulations of the kinetic ising model

4 (1993) 239

4 (1993) 339

4 (1993) 365

4 (1993) 345

4 (1993) 1147

4 (1993) 749

4 (1993) 1333

4 (1993) 357

4 (1993) 955

4 (1993) 969

4 (1993) 409

4 (1993) 993

4 (1993) 883

4 (1993) 1117

$4(1993) 1103$

4 (1993) 1233

4 (1993) 151

4 (1993) 1059

4 (1993) 1117

4 (1993) 1285

4 (1993) 919

$4(1993) 365$

$4(1993) 1137$

$\mathbf{N}$

Nakamura, A. see Stamatescu. I.O

Neuhaus, T. see Karsch, $F$.

4 (1993) 1233

4 (1993) 1195

o

Oberacker, V.E. see Wells, J.C.
Okabe, Y. see Ito, $N$.

4 (1993) 569

Okuda, M. see Stamatescu, I.O.

Ossadnik, P. Cellular automaton for the fracture of elastic media

4 (1993) 1233

$4(1993) 127$

$\mathbf{P}$

Panizzi, E. see Bartoloni, A

Panizzi, E. see Bartoloni, A.

Paolucci, P.S. see Bartoloni, A.

Paolucci, P.S. see Bartoloni, A.

Paolucci, P.S. see Bartoloni, A.

Paris, N. POMPC: A C language for data parallelism

Parisi, G. see Iori, G.

Pasini, P. see Chiccoli, C.

Pech, J. see Bartoloni, A.

Pech, J. see Bartoloni, A.

Pech, J. see Bartoloni, A.

Petiton, S. Saad, Y., Wu, K. \&

Ferng, W. Basic sparse matrix computations on the CM-5

Petkov, N. Fuzzy number sub traction convolution on the $C M-2$

Pic, M.M. \& Essafi, H. Wavelet transform on connection machine and sympati 2

Pili, P. A parallel raycast algorithm of CSG models on CM2

Place, D. see Mora, $P$

Plache, B. see Karsch, $F$

Planes, A. see Vives, $E$.

Porte, H. see Goedgebuer, J.-P.

Pöschel, T. see Buchholtz, V.

Potvin, J. see Chaara, A.M.

Prislan, O. see Wang, D.C.

Q

Quecke, G. Environments for parallel architectures

$4(1993) 121$

$\mathbf{R}$

Rajopadhye, S.V. Propagation of bores in incompressible fluids

Rapuano, F. see Bartoloni, A.

Rapuano, F. see Bartoloni, A.

Remiddi, E. see Caffo, $M$

Ritter, P. see Wang, D.C.

Ruffo, S. Chaos in coupled rotators

4 (1993) 955

4 (1993) 969

4 (1993) 955

4 (1993) 969

4 (1993) 993

4 (1993) 85

4 (1993) 1333

4 (1993) 1041

4 (1993) 955

4 (1993) 969

4 (1993) 993

4 (1993) 65

$4(1993) 181$

$4(1993) 97$

4 (1993) 29

4 (1993) 1059

4 (1993) 1195

4 (1993) 701

4 (1993) 299

4 (1993) 1049

4 (1993) 919

4 (1993) 151

$\mathbf{S}$

Saad, Y. see Petiton, S.

Sahimi, M. see Stauffer, $D$.

Sanz-Serna, J.M. \& Calvo, M.P.

Symplectic numerical methods for Hamiltonian problems

Sarno, R. see Bartoloni, A.

Sarno, R. see Bartoloni, A.
4 (1993) 621

4 (1993) 955

4 (1993) 969

4 (1993) 151

4 (1993) 375

4 (1993) 65

$4(1993) 401$

4 (1993) 385

4 (1993) 955

4 (1993) 969
4 (1993) 591 
Sarno, R. see Bartoloni, A.

Schaale, A. see Bel'kov, A.A.

Scheinine, A.L. Parallel computing at $\mathrm{CRS} 4$

Schilling, K. The connection machine in a university environment: Gesamthochschule Wuppertal

Schilling, K. \& Bali, G.S. The static quark-antiquark-potential: A 'classical' experiment on the connection machine $C M-2$

Schilling, K. see Bali, G.S

Schilling, K. see Bode, $A$

Scholl, H. see Alimi, J.-M.

Semeria, F. see Chiccoli, $C$

Sengotta, T. see Wang, D.C.

Shapira, S. see Adler, $J$.

Sharma, S.P. Assessment of nonequilibrium radiation computation methods for hypersonic flows

Shchur, L.N. see Talapov, A.L.

Singer Th, Husslein, J.M. see Hackl, $R$.

Smit, B. Hilbers, P.A.J. \& Esselink, K. Computer simula tions of surfactant self assembly

Smith, M. Dynamic load-balancing strategies for data parallel implementations of reactionevolution-migration systems

Soda, G. see Frasconi, P.

Speh, M. see Baker, $M$.

Stamatescu, I.O. Akemi, K., Fujisaki, M., Okuda, M., Tago, Y., Hashimoto, T., Hioki, S., Miyamura, O., Takaishi, T., Nakamura, A., de Forcrand. Ph. \& Hege, C. $Q C D$ on the massively parallel computer AP1000

Stauffer, D. \& Sahimi, M. High dimensional and very large cellular automata for immunological shape space

Steeb, W.-H. Fermi systems and computer algebra

Strayer, M.R. see Wells, J.C.

Strayer, M.R. see Dean, D.I

Strayer, M.R. see Dean, D.J.

Succi, S. Benzi, R. \& Massaioli,

$F$. A review of the lattice Boltzmann method

Svane, A. Electronic structure of solids in the self-interaction corrected local-spin-density approximation

$\mathbf{4}(1993) 993$
$\mathbf{4}(1993) 775$
$\mathbf{4}(1993) 1315$

$\mathbf{4}(1993) 137$

$\mathbf{4}(1993) 1167$
$\mathbf{4}(1993) 1179$
$\mathbf{4}(1993) 1205$
$\mathbf{4}(1993) 197$
$\mathbf{4}(1993) 1041$
$\mathbf{4}(1993) 151$
$\mathbf{4}(1993) 1007$

$\mathbf{4}(1993) 847$
$\mathbf{4}(1993) 787$
$\mathbf{4}(1993) 1117$

Tago, Y. see Stamatescu, I.O.

$4(1993) 1233$

Takaishi, T. see Stamatescu, I.O.

Talapov, A.L. Andreichenko, V.B., Dotsenko, V1.S. \& Shchur, L.N. First cluster algorithm special purpose processor

Tamayo, P. see Lomdahl, P.S.

Todesco, G.M. see Bartoloni, A.

Todesco, G.M. see Bartoloni, A.

Todesco, G.M. see Bartoloni, A.

Torelli, M. see Bartoloni, A

Torelli, M. see Bartoloni, A.

Torelli, M. see Bartoloni, A.

Trappenberg, T. see Moriarity, K.J.M.

Trew, A. Introduction to the Edinburgh parallel computing centre

Tripiccione, R. Dedicated computers for lattice gauge theory simulations

Tripiccione, R. see Bartoloni. A.

Tripiccione, R. see Bartoloni, A.

Tripiccione, R. see Bartoloni, A.

Tripiccione, R. APEIOO and beyond

4 (1993) 393

Tross, W. see Bartoloni, A.

Tross, W. see Bartoloni, A.

Tross, W. see Bartoloni, A.

4 (1993) 107

4 (1993) 17

4 (1993) 239

Ueberholz, P. see Bode, A.

Umar, A.S. see Wells, J.C.

$\mathrm{v}$

Velarde, M.G. Physics education: a survey of problems and possible solutions

Vicini, P. see Bartoloni, A.

Vicini, P. see Bartoloni, A.

Vicini, P. see Bartoloni, A.

Vinette, F. see $\breve{C} i \check{z} e k, J$.

Vives, E. \& Planes, A. Ordering kinetics by vacancies

$4(1993) 401$

4 (1993) 841

4 (1993) 459

4 (1993) 723

4 (1993) 1023

4 (1993) 409

von der Linden, W. What can we learn from $Q M C$ simulations

W

Wang, D.C. Möller, R., Carl, U., Prislan, O., Sengotta, T. \& Ritter, P. Parallel computer graphics at the institute of automation

Weber, M. Workstation clusters One way to parallel computing
$4(1993) 787$

4 (1993) 1075

4 (1993) 955

4 (1993) 969

4 (1993) 993

4 (1993) 955

4 (1993) 969

4 (1993) 993

$4(1993) 1285$

$4(1993)] 05$

4 (1993) 425

4 (1993) 955

4 (1993) 969

4 (1993) 993

4 (1993) 1323

4 (1993) 955

4 (1993) 969

4 (1993) 993

4 (1993) 1205

4 (1993) 459

4 (1993) 435

4 (1993) 955

4 (1993) 969

4 (1993) 993

4 (1993) 257

4 (1993) 701

4 (1993) 331

$4(1993) 151$

4 (1993) 1307 
Wells, J.C. Umar, A.S., Oberacker, V.E., Bottcher, C., Strayer, M.R., Wu, J.-S., Drake, J. \& Flanery, R. A numerical implementation of the Dirac equation on a hypercube multicomputer

Wells, J.C. see Dean, D.J.

Weniger, E.J. see Č́ ž žek, J.

Williams, A.R. Parallel computing and electronic-structure theory

Wittlich, T. see Lauwers, P.G.

$4(1993) 459$
$4(1993) 1023$
$4(1993) 257$
$4(1993) 445$
$4(1993) 609$

Wolff, U. High precision simulation techniques for lattice field theory

4 (1993) 451

Wu, J.-S. see Wells, J.C.

Wu, K. see Petiton, S.

4 (1993) 459

Y

Yeung, Y.Y. see Choy, T.L. $\quad 4$ (1993) 493

$\mathbf{Z}$

Zannoni, C. see Chiccoli, $C$.

Zeller, R. Green-function method for electronic structure of periodic crystals

4 (1993) 1041

4 (1993) 1109 